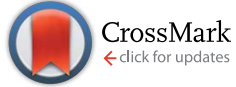

Cite this: Chem. Sci., 2014, 5, 4346

\title{
Charge transfer interactions in self-assembled single walled carbon nanotubes/Dawson-Wells polyoxometalate hybrids + t:
}

\author{
Concha Bosch-Navarro, ${ }^{a}$ Benjamin Matt, ${ }^{b}$ Guillaume Izzet, ${ }^{b}$ Carlos Romero-Nieto, $\S^{c}$ \\ Konstantin Dirian, ${ }^{c}$ Andrés Raya, ${ }^{d}$ Sergio I. Molina, ${ }^{d}$ Anna Proust, ${ }^{* b}$ Dirk M. Guldi, ${ }^{* c}$ \\ Carlos Martí-Gastaldo*a and Eugenio Coronado*a
}

\begin{abstract}
We demonstrate the success in self-assembling pyrene-modified Dawson-Wells-type polyoxometalates (POMs) with single walled carbon nanotubes (SWCNTs) by means of $\pi-\pi$ interactions. In this context, the immobilization of POMs onto SWCNTs is corroborated by aberration-corrected high-resolution electron microscopy, thermogravimetric analysis, and Raman spectroscopy. From steady-state and time-resolved photophysical techniques we derived evidence for mutual interactions between SWCNTs and POMs in the excited states. The latter are the inception to a charge transfer from the SWCNTs to the POMs. Our results corroborate the suitability of POM-SWCNTs assemblies for photoactive molecular devices.
\end{abstract}

Received 7th May 2014

Accepted 18th June 2014

DOI: $10.1039 / c 4 s c 01335 b$

www.rsc.org/chemicalscience

optoelectronics, ${ }^{4}$ energy storage, ${ }^{5}$ field-effect transistors (FETs $)^{6}$

\section{Introduction}

Tailoring hybrid nanostructures offers substantial advantages over the use of "classic" bulk solids as their use as buildingblocks may permit downscaling of the costs associated with more conventional technologies. ${ }^{1}$ Moreover, nanostructure design enables integrating molecular and bulk electronic structures into sophisticated architectures with variable degrees of complexity. The latter can be processed more easily and allows fine-tuning of the chemical and physical properties that arise at the nanoscale. In the context of nanoelectronics, single walled carbon nanotubes (SWCNTs) have emerged as ideal onedimensional wires on account of their unique electronic features, large specific surface areas, high mechanical strength, and remarkable chemical robustness. ${ }^{2}$ Hence, they have been implemented into practical devices in the areas of spintronics, ${ }^{3}$

\footnotetext{
aniversidad de Valencia (ICMol), Catedrático José Beltrán-2, Paterna, 46980, Spain. E-mail: eugenio.coronado@uv.es; carlos.marti@uv.es

${ }^{b}$ Institut Parisien de Chimie Moléculaire, Sorbonne Universités UPMC Univ Paris 06, UMR CNRS 7201, Université Pierre et Marie Curie, 4 place Jussieu, Case 42, 75252, Paris Cedex 05, France. E-mail: anna.proust@upmc.fr

'Friedrich-Alexander-Universität Erlangen-Nürnberg, Department Chemie und Pharmazie und Interdisziplinäres Zentrum für Molekulare Materialien, Egerlandstrasse 3, 91058, Erlangen, Germany. E-mail: dirk.guldi@fau.de

${ }^{d}$ Departamento de Ciencia de los Materiales e I.M. y Q.I., Facultad de Ciencias, Universidad de Cádiz, Campus Río San Pedro, s/n, Puerto Real, 11510, Cádiz, Spain $\dagger$ Electronic supplementary information (ESI) available: Thermogravimetric analysis, additional HRTEM, HAADF-STEM and BF-STEM images, and differential absorption spectra. See DOI: 10.1039/c4sc01335b

$\$$ In memory of Prof. Mark Elliot.

$\S$ Current address: Organisch-Chemisches Institut, Im Neuenheimer Feld 270, 69129, Heidelberg, Germany.
}

or sensors. ${ }^{7}$

Polyoxometalates (POMs) are an archetypical family of inorganic polynuclear metal-oxo nanoclusters. ${ }^{8}$ Their unmatched structural versatilities provide accessible chemical means to modulate their electronic behavior. This results in a rich landscape of magnetic, catalytic, and redox properties, which, alongside their chemical robustness, are currently attracting particular interest in areas like molecular magnetism, ${ }^{9}$ molecular spintronics, ${ }^{10}$ and water oxidation catalysis. ${ }^{11}$

This interest has now evolved into the creation of SWCNTPOM hybrid architectures by immobilizing electronically active POMs onto the sidewalls of carbon nanotubes by means of either covalent or non-covalent interactions. For example, Chen et al. reported the grafting of the Keggin-type $\mathrm{H}_{7} \mathrm{PMo}_{12} \mathrm{O}_{42}$ onto SWCNTs and the use of the resulting assemblies as a catalyst support for the dispersion of electrocatalytic nanoparticles. ${ }^{12}$ Mallah et al. succeeded in isolating magnetic hybrids by incorporating magnetic POMs like $\left[\mathrm{Fe}_{4}\left(\mathrm{H}_{2} \mathrm{O}\right)_{2}\left(\mathrm{FeW}_{9} \mathrm{O}_{34}\right)_{2}\right]^{10-}$ or $\left[\mathrm{As}_{2} \mathrm{~W}_{20} \mathrm{O}_{68} \mathrm{Co}\left(\mathrm{H}_{2} \mathrm{O}\right)\right]^{8-}$ and suggested that the magnetic response of the clusters was retained after their grafting. ${ }^{13}$ More recent reports illustrate the potential benefits of these assemblies in catalysis ${ }^{14}$ or molecular cluster batteries. ${ }^{15}$ Whilst these precedents solely explored simple chemisorption or electrostatic trapping of the POMs to immobilize them on the surface of the chemically unmodified nanotubes, there are also abundant examples of organic-inorganic POM-based hybrids assembled from covalent post-functionalization reactions of interest in artificial photosynthesis, ${ }^{16}$ molecular self-healing ${ }^{17}$ or molecular electronics. ${ }^{18,19}$ The covalent approach renders stronger interactions between the organic and the inorganic 
components besides better control over the structure of the hybrids. $^{20}$

Although covalent or electrostatic interactions provide strong SWCNT-POM coupling, they also affect the electronic properties of the nanotubes as a result of the introduction of defects to the original $\mathrm{sp}^{2}$ structure. ${ }^{21}$ Supramolecular $\pi-\pi$ grafting affords a strong contact within the carbon-based hybrids, whilst leaving the electronic properties of SWCNTs intact, featuring as the best choice for the fabrication of photoactive assemblies. In particular, pyrene (pyr) and pyr derivatives enable strong interactions within carbon-based hybrids without affecting the electronic properties of SWCNTs, including ballistic conductance and optical transitions. Previous studies have demonstrated the versatility of this polyaromatic hydrocarbon to facilitate the grafting of a wide variety of molecular building blocks like redox-active transition metal complexes, ${ }^{22}$ single molecule magnets, ${ }^{23}$ phthalocyanines, ${ }^{24}$ quantum dots, ${ }^{25}$ and even DNA to the sidewalls of SWCNTs. ${ }^{26}$

Built upon these considerations, the use of POMs derivatized with pyr as anchoring groups could give rise to stable hybrids, whose stability might be further increased by a nanotweezer-like geometry (i.e. when two or more aromatic units are involved in the binding with the SWCNTs). ${ }^{27}$ The strong coupling between pyr and the nanotubes' sidewalls is also favorable to study the electronic communication within the hybrid. However, noncovalent functionalization of SWCNTs with POMs has been mostly unexplored to date - only two examples have been reported $^{28}$ - likely due to the intrinsic synthetic difficulties to chemically derivatize the POM's periphery. Among the wide array of available POMs, we targeted Dawson-Wells polyoxometalates, $\left[\mathrm{M}_{18} \mathrm{O}_{62} \mathrm{X}_{2}\right]^{n-},{ }^{29}$ on the basis of their chemical versatility. The latter permits modification of the inorganic framework to produce non-classical or lacunary Dawson-Wells POMs ${ }^{30}$ as well as more complex multifunctional materials and hybrids. ${ }^{20}$

In this work, we report the preparation, characterization and electronic communication in photoactive SWCNT-POM hybrids, which incorporate Dawson-Wells-type POMs bearing pendant pyrenes (POM-pyr; Fig. 1) to enable their grafting onto SWCNTs via $\pi-\pi$ interactions. POM-pyr was designed to feature a nanotweezers-like geometry that reinforces the coupling between the two anchoring pyrenes and SWCNTs to maximize the stability of the resulting hybrids. ${ }^{27}$

Our photophysical investigations suggest that the use of fully conjugated organic moieties grafted to the POMs is decisive to trigger an efficient electronic communication between the different constituents, with SWCNTs and POMs acting as electron donors and acceptors, respectively. Combination of these features with the ability of POMs to undergo multiple reductions renders the described SWCNT-POM hybrids as versatile electron reservoirs for catalysis.

\section{Results and discussion}

\section{Synthesis of SWCNT-POM hybrids}

The synthesis of the tetrabutylammonium salt of the pyrenederivatized Dawson-Wells polyoxometalate (TBA) $)_{6}\left[\mathrm{P}_{2} \mathrm{~W}_{17} \mathrm{O}_{61}\right.$ $\left.\left\{\mathrm{O}(\mathrm{Si} \text {-phenyl-ethynyl-pyrene })_{2}\right\}\right]$ (POM-pyr; Fig. 1) was prepared

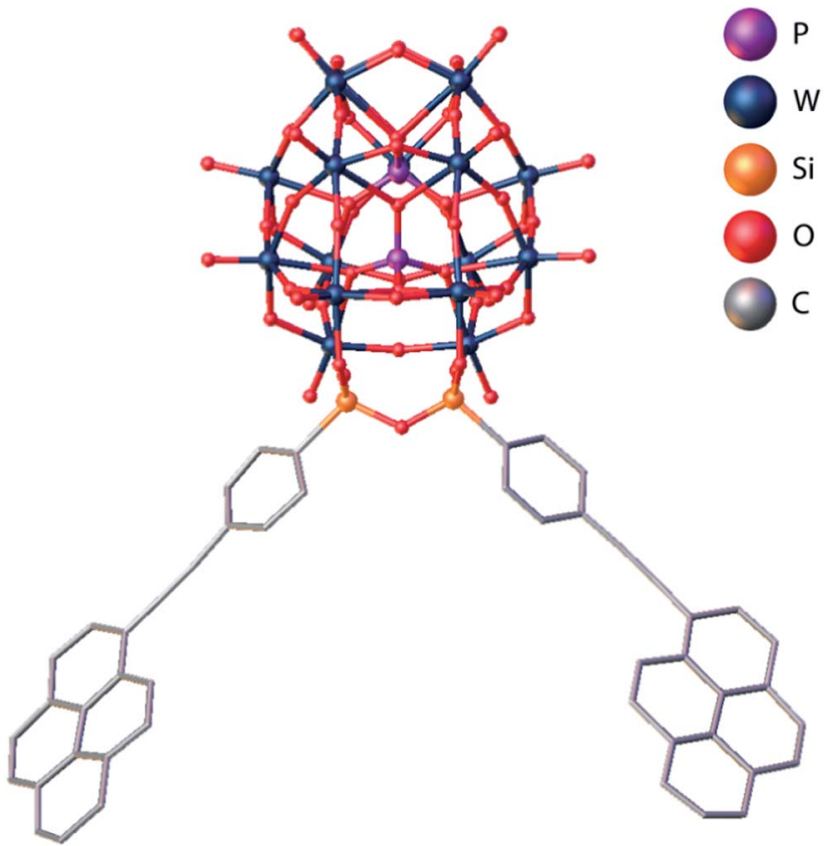

Fig. 1 Structure of $\left[\mathrm{P}_{2} \mathrm{~W}_{17} \mathrm{O}_{61}\left\{\mathrm{O}(\mathrm{Si} \text {-phenyl-ethynyl-pyrene })_{2}\right\}\right]^{6-}$ (POM-pyr).

as previously described by Sonogashira cross-coupling reaction of an iodo aryl POM intermediate with an alkynyl pyrene derivative. ${ }^{31}$ The SWCNT-POM hybrids are prepared by adding POM-pyr to a fresh dispersion of commercial grade SWCNTs (i.e., HiPco ${ }^{\circledR}$ and Elicarb ${ }^{\circledR}$ SWCNTs) in DMF, which is left to settle overnight (Scheme 1). The residue is then collected by centrifugation and filtration, followed by thorough washing with DMF and acetonitrile to ensure removal of the excess of non-grafted POM-pyr.

\section{Thermogravimetric analysis (TGA)}

TGA was firstly used to confirm the functionalization of SWCNTs (Fig. SI $1 \dagger$ ). Thermal decomposition of the hybrid is

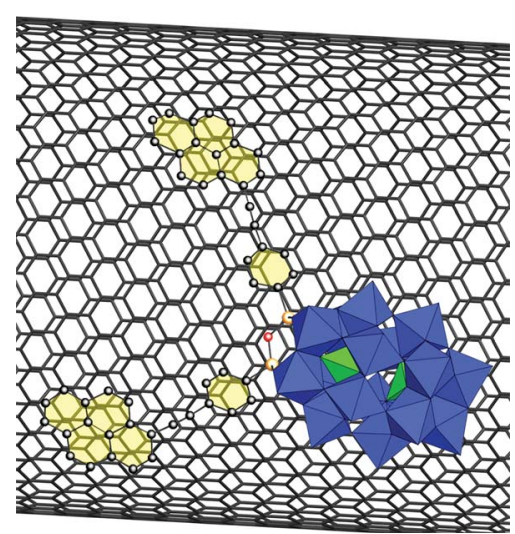

Scheme 1 SWCNT-POM formed upon assembly of POM-pyr by means of $\pi-\pi$ stacking of the pendant pyrenes of POM-pyr onto the sidewalls of SWCNTs. Tetrabutylammonium (TBA) cations are omitted for clarity. 
dominated by the nanotubes' response. Bare SWCNTs oxidize in a single, narrow process centered at $580{ }^{\circ} \mathrm{C}$, whereas SWCNTPOM displays, on one hand, in the temperature regime up to $500{ }^{\circ} \mathrm{C}$ a thermal decomposition at $450{ }^{\circ} \mathrm{C}$ and, on the other hand, in the temperature regime beyond $500{ }^{\circ} \mathrm{C}$ a similar thermal stability to SWCNTs. As such, this behavior is governed by the presence of immobilized POM-pyr, whose organic moieties and ammonium counterions start to decompose at $350{ }^{\circ} \mathrm{C}$ for the starting TBA salt. The latter does not exert significant damage to the SWCNT structure, which is generally associated to poorer thermal stability due to the introduction of defects. ${ }^{32}$ According to the calculations described in Fig. SI1, $\dagger$ the remaining mass of the SWCNT-POM hybrids with respect to the pristine SWCNTs is estimated to be close to 10\% (1 POM-pyr unit per 4600 carbon atoms). ${ }^{33}$

\section{Electron microscopy and EDS (energy-dispersive X-ray spectroscopy) analysis}

High Resolution Transmission Electron Microscopy (HRTEM) images were collected from samples prepared by drop casting fresh EtOH suspensions of SWCNT-POM hybrids on a carboncoated copper grid. Fig. SI $2 \dagger$ illustrates the presence of nanotube bundles with multiple interlocked junctions, which are mainly composed by carbon according to local EDS analysis, alongside a heterogeneous distribution of conglomerates with higher contrast composed of $\mathrm{Si}$ and $\mathrm{W}$, consistent with the immobilization of Dawson-Wells-type POMs.

We next performed aberration-corrected High-Angle Annular Dark-Field Scanning-Transmission Electron Microscopy (HAADF-STEM) and Bright Field (BF)-STEM to determine the orientation of the POM-pyr clusters along the surface of SWCNTs (Fig. 2 and SI $\dagger \dagger$ ). The major advantage of HAADFSTEM over HRTEM is the non-existence of contrast reversal with specimen thickness and microscope parameters. This is due to the incoherent nature of the HAADF-STEM image process formation. Sub-Ångstrom resolution is achieved by using aberration corrected electron microscopes and, therefore, atomic columns and even individual atoms can be imaged. The intensity in HAADF images can be correlated with the atomic number $(Z)$ of the present elements. Hence, elements with a high $Z$ such as tungsten $(\mathrm{W} ; Z=74)$ show high contrast, whereas lighter elements like carbon $(C ; Z=6)$ are hardly discernable. HAADF-STEM in Fig. 2 displays the presence of bright spots decorating the sidewalls of SWCNTs. These bright spots correspond to the projection of the structure formed by the $\mathrm{W}$ atoms of POMs. However, it remains difficult to establish exact atom-by-atom correlation between the observed projection and the structure of POM-pyr. The following aspects need careful consideration. On one hand, from analyzing just a single image we cannot differentiate between a bright spot that corresponds to a single atom or to two atoms, which are aligned or partially overlapped in the direction of the optical axis. On the other hand, several orientations of the SWCNT-POM hybrids may result in similar projections relative to the plane perpendicular to the incident electron beam.
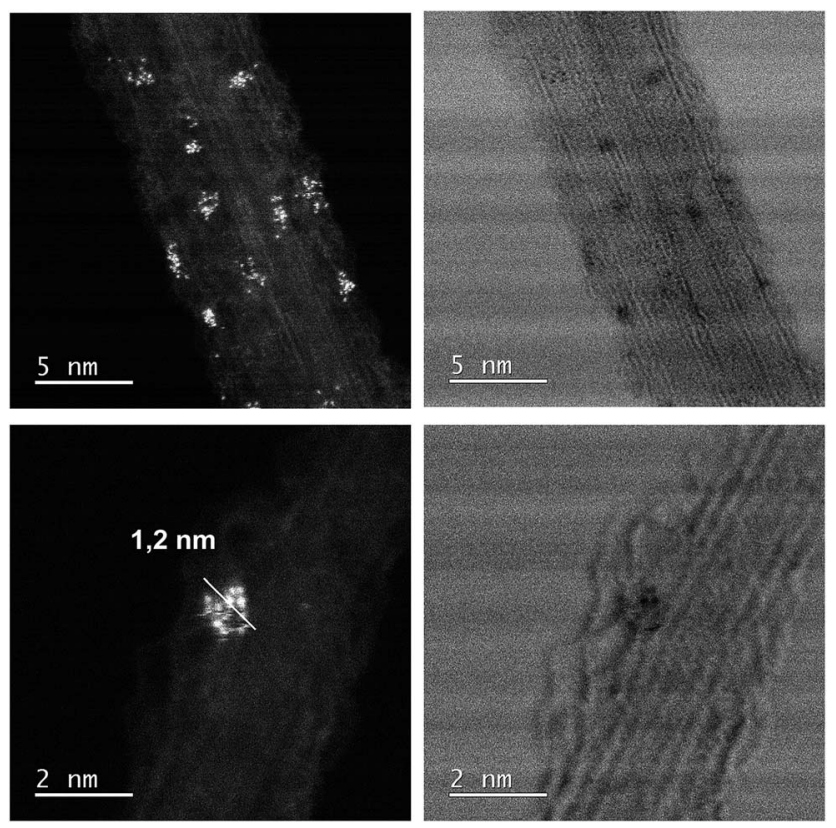

Fig. 2 HAADF-STEM (top left) and BF-STEM (top right) images showing the presence of POM-pyr clusters (bright spots) along the sidewalls of SWCNTs. In the bottom images, a zoom-in is done over a bright spot, which has a size of $1.2 \times 1 \mathrm{~nm}$, in good agreement with the size of the POM-pyr clusters.

Electron tomography experiments could not be performed because of the instability of the POM structure caused by the interaction with the electron beam. Besides, SWCNTs are more easily distinguished in the $\mathrm{BF}$ image (Fig. 2, right). The latter confirms the heterogeneous distribution of objects along SWCNTs composed of species with heavy atoms and with sizes of approximately $1.2 \mathrm{~nm}$, in excellent agreement with the dimensions of the Dawson-Wells-type POMs.

\section{X-Ray photoelectron spectroscopy (XPS)}

XPS was also used to determine the presence of POM-pyr in SWCNT-POM hybrids and to rule out changes in its chemical nature upon immobilization. According to Fig. 3, the binding energies (BEs) for the $\mathrm{W}$ 4f signals in the XPS spectrum of SWCNT-POM hybrids agree with the data collected from a crystalline sample of POM-pyr. Both spectra display a doublet centered at ca. 35 and $38 \mathrm{eV}$, with an energy splitting of approximately $2.1 \mathrm{eV}$. These peaks originate from the spin-orbit splitting into the $\mathrm{W} 4 \mathrm{f}_{7 / 2}$ and $\mathrm{W} 4 \mathrm{f}_{5 / 2}$ levels, respectively (Table 1). ${ }^{34}$ In summary, these values confirm the presence of $\mathrm{W}-\mathrm{O}$ bonds in both samples and confirm the presence of intact POM-pyr in SWCNT-POM hybrids.

\section{Physicochemical properties}

Upfront we tested POM-pyr and its relevant references in oxidative and reductive processes - a full account is given in ref. 16 and 31. Given that standard electrochemical measurements, like cyclic voltammetry, etc. are reported in the aforementioned papers, we focused our investigation onto 


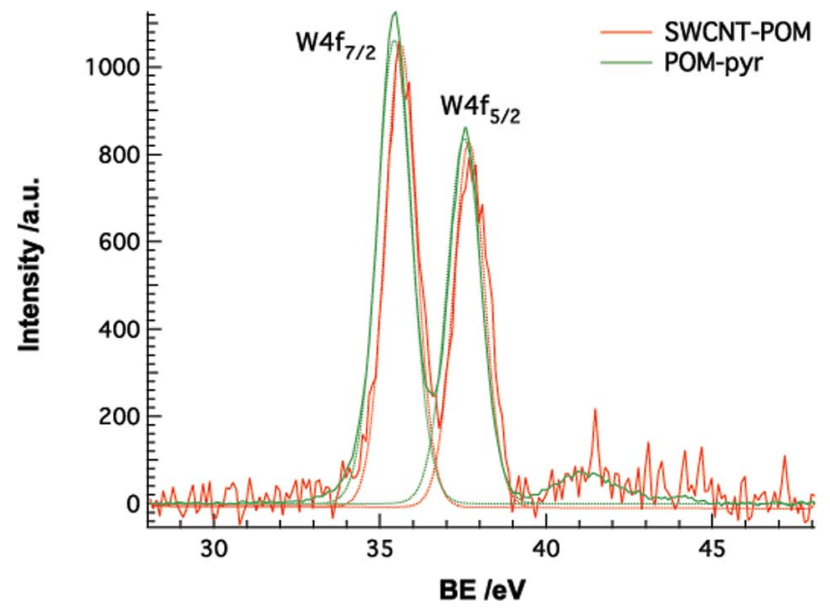

Fig. 3 W 4 f high-resolution XPS spectra of POM-pyr (green spectrum) and SWCNT-POM (red spectrum). The dashed-lines represent the deconvolution for each sample.

Table 1 Binding energies (BE) extracted from the XPS spectra of POMpyr and SWCNT-POM

\begin{tabular}{lll}
\hline \multirow{2}{*}{ Sample } & $\mathrm{W} 4 \mathrm{f} / \mathrm{BE}, \mathrm{eV}$ & \\
\cline { 2 - 3 } & $\mathrm{W} 4 \mathrm{f}_{7 / 2}$ & $\mathrm{~W} 4 \mathrm{f}_{5 / 2}$ \\
\hline POM-pyr & 35.43 & 37.53 \\
SWCNT-POM & 35.62 & 37.68
\end{tabular}

spectroelectrochemical measurements. In particular, we applied variable potentials in either the reductive or the oxidative ranges and recorded the absorption spectra in given intervals. Notably, differential spectra rather than absolute spectra are shown to illustrate the spectral changes. Thus, in POM-pyr the two first reductions $(-0.73 \mathrm{~V}$ and $-1.14 \mathrm{~V} v s$. SCE) are POM centred, while the oxidation ( $c a .1 .3 \mathrm{~V} v s$. SCE) involves pyr. In terms of differential absorption changes, applying a potential of $+0.9 \mathrm{~V} v \mathrm{~s}$. $\mathrm{Ag} / \mathrm{AgNO}_{3}$ has been shown to oxidize quasi-reversibly pyr and pyrene-1-methanol, which due to its functionalization serves as a reference to POM-pyr in the form of newly developing minima at 308,322 , and $336 \mathrm{~nm}$ for the earlier as well as 315,328 , and $344 \mathrm{~nm}$ for the latter in $\mathrm{TBAPF}_{6}$ containing DMF solutions (either 0.1 or $0.2 \mathrm{M}$ ) (Fig. SI4†). These are accompanied by maxima at 360, 400, 430, and $457 \mathrm{~nm}$ for pyr and at 362, 375 and $396 \mathrm{~nm}$ for pyrene-1methanol. In the case of POM-pyr, where the oxidation leads to irreversible changes, a bleaching of the sharp maxima at 300 , 369 , and $392 \mathrm{~nm}$ occur, which are accompanied by broad and still defined features throughout the $400-500 \mathrm{~nm}$ range (Fig. SI4†).

In contrast, when applying a potential of $-0.8 \mathrm{~V} v s$. Ag-wire as a pseudo reference electrode, discernible changes can be observed, which are related to the filling of electronic d-states, and includes a sharp and a broad maximum between 400 and $550 \mathrm{~nm}$ and between 600 and $1200 \mathrm{~nm}$, respectively (Fig. 4). The corresponding maxima are noted at 450 and $830 \mathrm{~nm}$. Upon

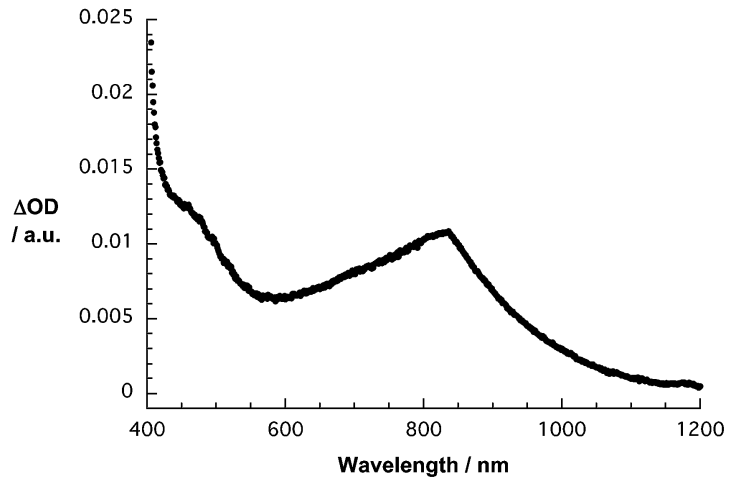

Fig. 4 Differential absorption spectra (visible and near-infrared) obtained upon electrochemical reduction of POM-pyr in DMF with an applied potential of $-0.8 \mathrm{~V}$ vs. Ag-wire as a pseudo reference electrode.

applying a moderate positive potential $(0.1 \mathrm{~V})$, the aforementioned features retransform to those of the ground state. This trend corroborates previous cyclic voltammetric investigations in terms of the reversibility of the underlying process.

Insights into electronic communication between POM-pyr and SWCNTs were carefully probed by means of steady state and transient absorption assays. DMF suspensions of SWCNT-POM hybrids were initially tested by absorption spectroscopy and compared to the response of bare SWCNTs, which were suspended with sodium dodecyl benzene sulfonate (SWCNT/SDBS) in $\mathrm{D}_{2} \mathrm{O}$. Here, two types of SWCNTs, namely HiPco ${ }^{\circledR}$ and Elicarb ${ }^{\circledR}$ SWCNTs, stood at the forefront of the investigations. Owing, however, to the lack of notable absorption/fluorescence features for Elicarb ${ }^{\circledR}$ SWCNTs in the NIR - either in the form of SWCNT-POM hybrids or SWCNT/SDBS - we focused our investigations on HiPco SWCNTs. A close look at the absorption spectra of HiPco SWCNT-POM in DMF and HiPco SWCNT/ SDBS in $\mathrm{D}_{2} \mathrm{O}$ reveals $\mathrm{M}_{11}, \mathrm{E}_{22}$, and $\mathrm{E}_{11}$ absorption peaks at 420, $450,510,560,600,660,740,1010,1190,1325$, and $1450 \mathrm{~nm}$ (Fig. 5). ${ }^{35,36}$ The absence of appreciable shifts, which are also

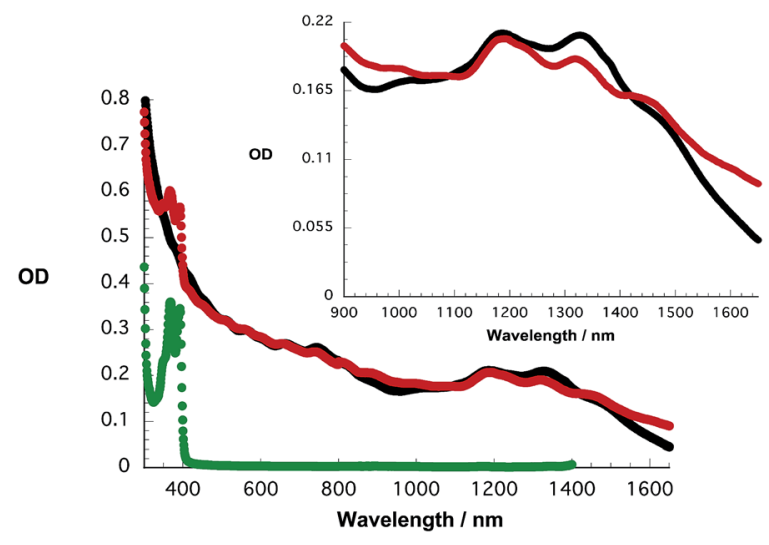

Fig. 5 Absorption spectra of SWCNT/SDBS in $\mathrm{D}_{2} \mathrm{O}$ (black spectrum), SWCNT-POM in DMF (red spectrum), and POM-pyr in DMF (green spectrum). Inset illustrates the near-infrared part of the absorption spectra. 
seen in the absence of POM-pyr, between the earlier and the latter, leads us to rule out the presence of electronic interactions in the ground state of HiPco SWCNT-POM hybrids. Previous studies have revealed red shifts of up to $30 \mathrm{~nm} .{ }^{37}$ It is worthwhile highlighting that DMF solutions of POM-pyr present a shoulder at $350 \mathrm{~nm}$ and maxima at 368 and $392 \mathrm{~nm}$ in their absorption spectra. Similarly, the absorption spectrum of HiPco SWCNT-POM displays maxima at 350, 368 and $390 \mathrm{~nm}$, which are, therefore, attributed to the presence of POM-pyr that are immobilized onto HiPco.

Next, the fluorescence features of HiPco SWCNT/SDBS and HiPco SWCNT-POM hybrids were investigated. In particular, HiPco SWCNT-POM display $\mathrm{E}_{11}$ fluorescence maxima at 1076, 1127, 1212, 1295, and $1437 \mathrm{~nm}-(10,2),(9,4),(8,6),(8,7)$ and $(9,7)$ SWCNTs - when exciting at $650 \mathrm{~nm}$ (Fig. 6). Owing to the fact that the fluorescent features of HiPco SWCNT-POM hybrids mirror image the corresponding ground state absorption, we associate both processes to the fundamental transitions across the band gap. Contrasting the fluorescence of the SWCNT-POM hybrids with that of SWCNT/SDBS at equal absorbances at the excitation wavelength sheds light onto the mutual interactions via either radiative or non-
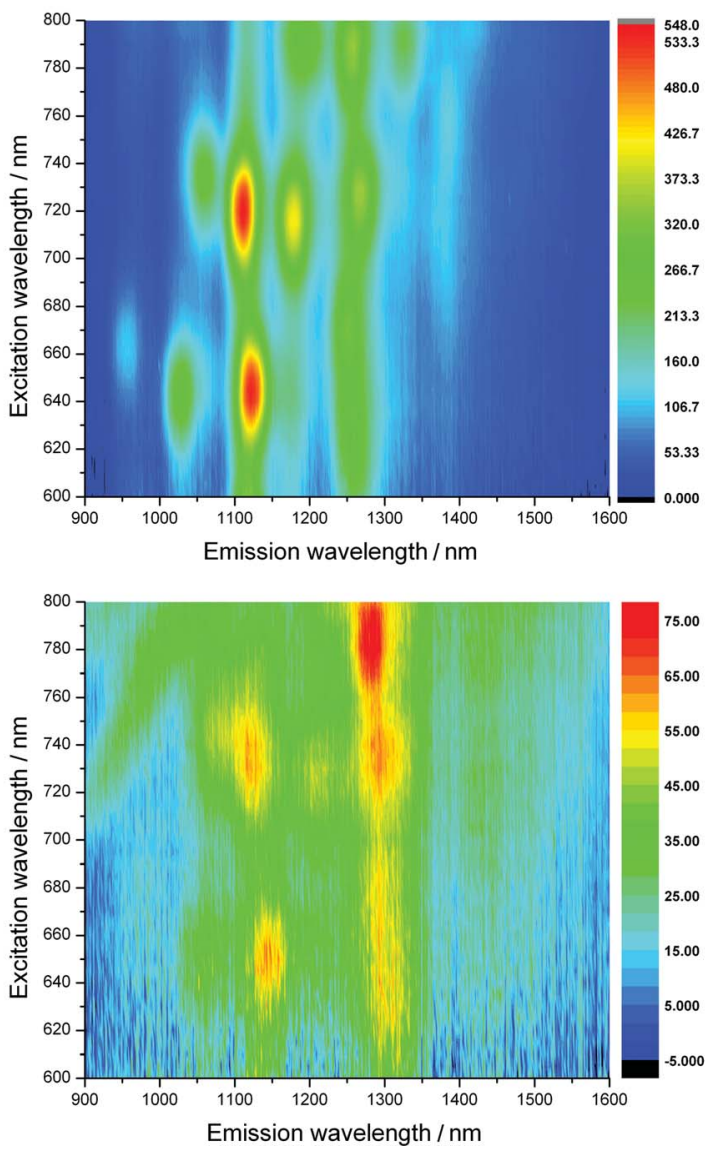

Fig. 6 (Top) 3D steady-state NIR fluorescence spectrum with increasing intensity from blue to green to yellow and to red of SWCNT/ SDBS in $D_{2} \mathrm{O}$. (Bottom) 3D steady-state NIR fluorescence spectrum with increasing intensity from blue to green to yellow and to red of SWCNT-POM in DMF radiative decays in the SWCNT-POM hybrids (Fig. 6 and Fig. 7). To this end, HiPco SWCNT-POM hybrids reveal an energetic shift of the fluorescent transitions in the form of redshifted maxima relative to those seen for HiPco SWCNT/SDBS. For HiPco SWCNT/SDBS, maxima at 1054, 1113, 1174, 1268, and $1376 \mathrm{~nm}$ were measured, which correspond to $(10,2),(9,4)$, $(8,6),(8,7)$, and $(9,7)$ SWCNTs, respectively. ${ }^{36}$

We rationalize a redistribution of electron density from SWCNTs to the electron-accepting POM-pyr to be responsible for the noted shift to lower energies. Examination of the fluorescence intensities (Fig. 7) prompts to approximately $90 \%$ quenching of the HiPco SWCNTs features. Regarding the electron accepting and electron donating features of POMpyr and SWCNTs, respectively, we hypothesize that the fluorescence quenching is due to a non-radiative singlet excited state deactivation in the form of an electron transfer process from the photoexcited SWCNT to the POM with energies of equal or less than $1.2 \mathrm{eV}$ (Fig. 8). ${ }^{16,37}$ The relative excited state energies of SWCNTs and POMs were determined based on the long wavelength absorption and short wavelength fluorescence as $\sim 1.2$ and $\sim 3.0 \mathrm{eV}$, respectively. These contradict, however, the feasibility of an energy transfer, which would be thermodynamically uphill. Moreover, we have no particular evidence for an energy transfer between different SWCNTs. The latter is taken from the fact that the fluorescence of $(10,2),(9,4),(8,6),(8,7)$, and $(9,7)$ SWCNTs are equally quenched.

Electronic interactions in HiPco SWCNT-POM hybrids were corroborated by Raman experiments. Here, the most important SWCNT signatures, which are RBM $\left(\sim 300 \mathrm{~cm}^{-1}\right), \quad \mathrm{D}$ $\left(\sim 1300 \mathrm{~cm}^{-1}\right), \mathrm{G}\left(\sim 1600 \mathrm{~cm}^{-1}\right)$, and $2 \mathrm{D}$ modes $\left(\sim 2550 \mathrm{~cm}^{-1}\right),{ }^{28}$ reveal upshifts in $\mathrm{D}_{2} \mathrm{O}$ suspensions of HiPco SWCNT-POM with respect to HiPco SWCNT/SDBS as well as in the solid without evidencing loss in resonance. For example, the $\mathrm{G}$ and $2 \mathrm{D}$ modes of HiPco SWCNTs shift from $1590 \pm 2$ to $1592 \pm 2 \mathrm{~cm}^{-1}$ and from $2548 \pm 2$ to $2552 \pm 2 \mathrm{~cm}^{-1}$, respectively (Fig. 9). This leaves no doubt that interactions are indeed operative between SWCNTs and POM-pyr but only in the excited state.

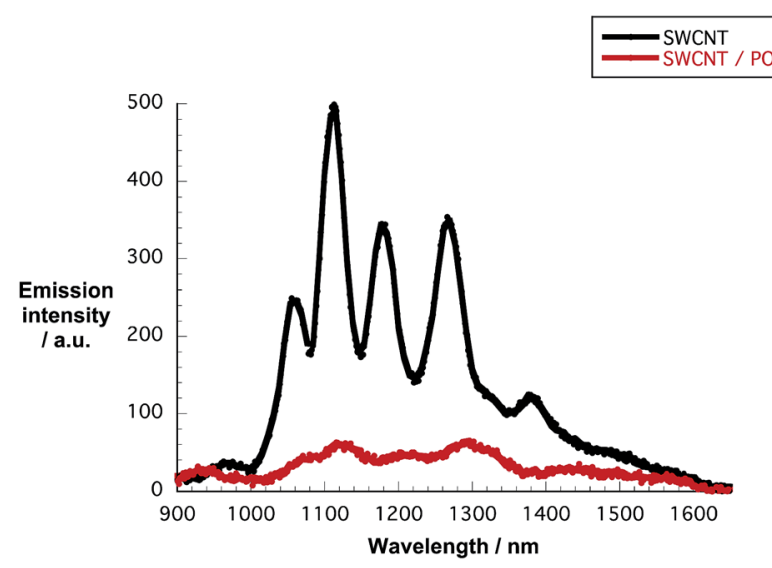

Fig. 7 Comparison of the NIR fluorescence spectra of SWCNT/SDBS (black spectrum) in $\mathrm{D}_{2} \mathrm{O}$ and SWCNT-POM (red spectrum) in DMF $650 \mathrm{~nm}$ excitation. 


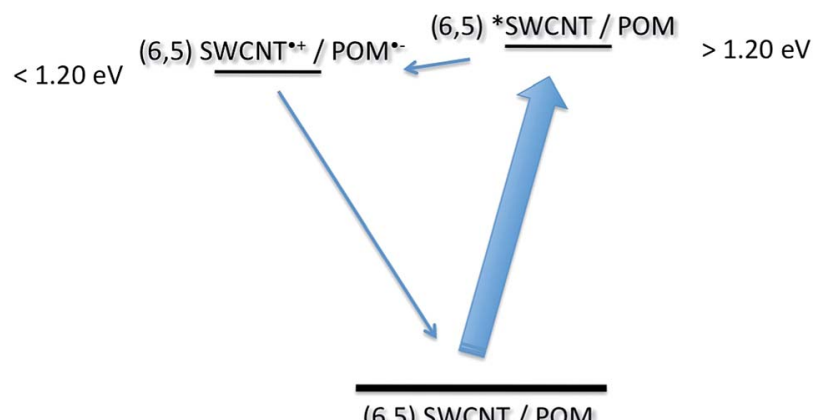

$(6,5)$ SWCNT / POM

Fig. 8 Energy diagram illustrating the charge separation evolving from photoexcited SWCNTS - exemplified for $(6,5)$ SWCNT to POM and subsequent charge recombination in competition with radiative and non-radiative (not shown) deactivation.
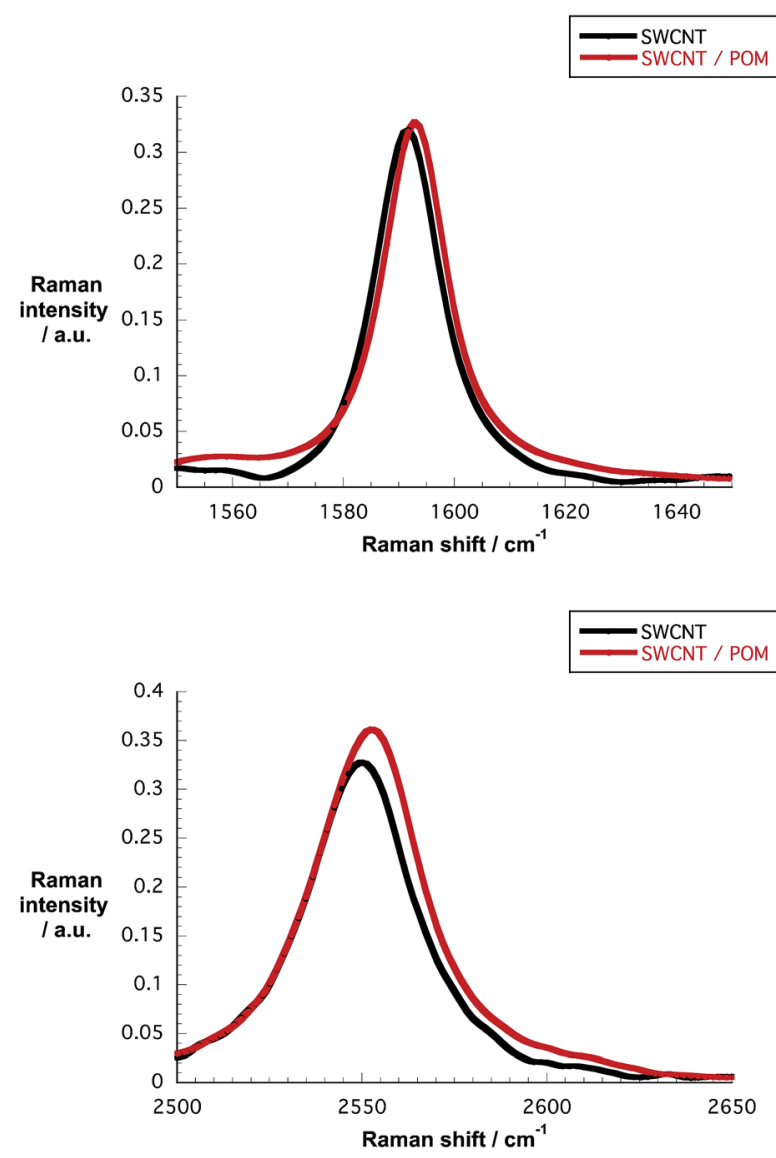

Fig. 9 (Top) Raman spectra of SWCNT/SDBS (black spectrum) in $\mathrm{D}_{2} \mathrm{O}$ suspension and SWCNT-POM (red spectrum) in DMF showing the G band. (Bottom) Raman spectra of SWCNT/SDBS (black spectrum) in $\mathrm{D}_{2} \mathrm{O}$ suspension and SWCNT-POM (red spectrum) in DMF showing the $D^{*}$ band. The spectra have been taken upon $1064 \mathrm{~nm}$ excitation.

Augmentation of electronic interactions in the ground state through, for example, charge transfer is ruled out, since no additional features are superimposed on the $\mathrm{G}$ band.

To determine the dynamics of the ultrafast excited state deactivation in HiPco SWCNT-POM hybrids, which was inferred from the fluorescence assays (vide supra), we employed transient absorption spectroscopy. In reference experiments, photoexcitation of POM-pyr (Fig. SI5 $\dagger$ ) and HiPco SWCNT/SDBS (Fig. SI6†) were probed at $387 \mathrm{~nm}$. For POM-pyr, centered excited states are generated instantaneously upon photoexcitation. The corresponding spectral characteristics include transient maxima at 525, 630, 700, 1130, and $1350 \mathrm{~nm}$ and are assigned to higher lying excited states. In line with this assignment is the fact that an overall short lifetime of $2 \pm 0.2$ ps transforms these into maxima at 515, 600, 920, 1090, and $1290 \mathrm{~nm}$. From here, we note a biphasic decay. On one hand, after a short lifetime of around $75 \pm 25$ ps maxima are noted at $485,535,600$, and $660 \mathrm{~nm}$. The short lifetime is rationalized on the basis of strong spin-orbit couplings due to the presence of the metal centres and, in turn, correlates with the decay of the singlet excited state. On the other hand, a long lifetime of $>7500 \mathrm{ps}$, which relates to the correspondingly formed triplet excited state, renders this transient rather stable prior to undergoing a possible electron transfer. With respect to the HiPco SWCNT/SDBS, the baseline is replaced with a strong bleaching that dominates the differential absorption spectra throughout the visible and near-infrared regions, where absorptive transitions of semiconducting SWCNTs appear, respectively (Fig. SI6†). The major minima are seen at 975, 1190, 1325, and $1460 \mathrm{~nm}$. Multiwavelength analyses of the bleaching characteristics in the near-infrared resulted in complex dynamics with two dominant lifetimes, namely $1.2 \pm$ 0.2 and $520 \pm 10$ ps. Throughout these multiexponential decays the original absorption/baseline is quantitatively reinstated. Hereby, the polydisperse nature of HiPco SWCNTs evokes a superimposition of a series of bleaching features. The latter is correlated with individual SWCNTs that absorb in nearly the same energetic range.

When turning to the HiPco SWCNT-POM hybrids, conditions were chosen that guaranteed stable SWCNT suspensions. Interesting is the fact, that the instantaneous bleaching follows the trend seen in the absorption assays. To this end, minima and shoulders in the differential absorption spectra at $565,605,660,995,1193,1320,1445$, and $1585 \mathrm{~nm}$ correlate well with the maxima and shoulders found in the absorption spectra at 560, 605, 660, 1194, 1320, 1445, and $1585 \mathrm{~nm}$ and, in turn, are SWCNT centered (Fig. 10). In addition, maxima are noted at 475, 495, 535, and $820 \mathrm{~nm}$. In fact, lifetimes of 25 \pm 4 and $1600 \pm 100$ ps were derived from a multiwavelength analysis, which strongly evoke charge separation and charge recombination. Evidence for the charge transfer hypothesis, that is, charge separation and charge recombination, was learnt from fine structured maxima in the visible and the near-infrared at 475, 525, 585, 615, 690, 1255, 1375, and 1525 $\mathrm{nm}$, which feature weak positive differential absorption changes. In addition, broad maxima are discernable in the 700 to $1200 \mathrm{~nm}$ range, in general, and the $850 \mathrm{~nm}$ maximum, in particular. In agreement with previous spectroelectrochemical experiments, we assign the earlier to oxidized SWCNTs, while the latter bears great resemblance with reduced POM-pyr. 

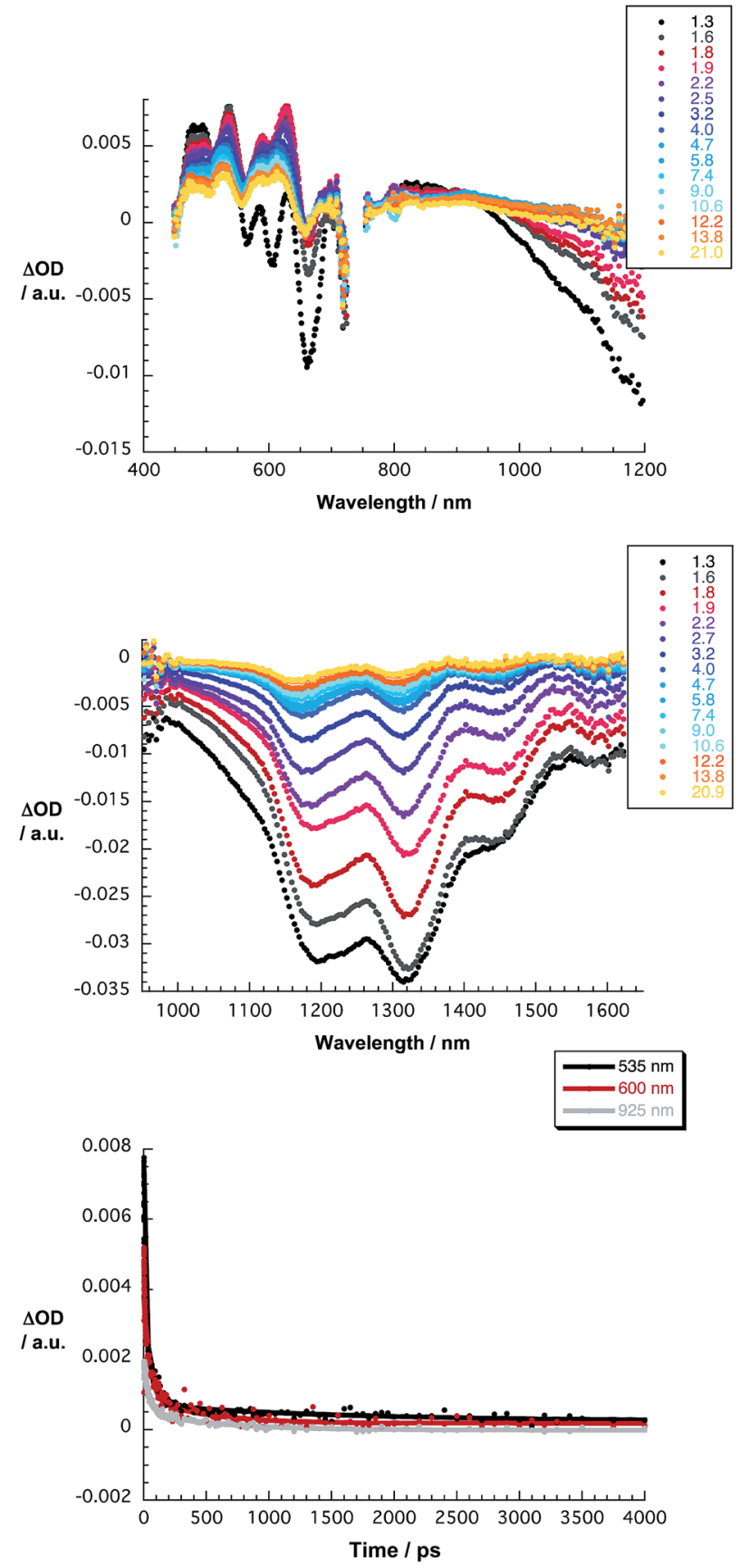

Fig. 10 (Top) Differential absorption spectra (visible and near infrared) obtained upon femtosecond pump probe experiments $(387 \mathrm{~nm})$ of SWCNT-POM in DMF with time delays of 1.3 and 20.9 ps at room temperature. (Centre) Differential absorption spectra (extended near infrared) obtained upon femtosecond pump probe experiments $(387 \mathrm{~nm})$ of SWCNT-POM in DMF with time delays of 1.3 and $20.9 \mathrm{ps}$ at room temperature. (Bottom) Time absorption profiles of the spectra shown in the upper part at 535 (black spectrum), 600 (red spectrum), and $925 \mathrm{~nm}$ (grey spectrum) monitoring the charge transfer.

\section{Experimental}

\section{Synthesis}

General synthetic remarks. Elicarb Single Walled Nanotubes (SWCNTs) were purchased from Thomas Swan Co. Ltd. and
HiPco SWCNTs were purchased from Unidym Inc. The samples were filtered employing a vacuum filter funnel pore size 3 , and nylon membrane filters ( $ø 0.2 \mu \mathrm{m}$ pore size). The ultrasound conditions were achieved with a Brandson 5510E-MT operating at $135 \mathrm{~W}$.

Synthesis of POM-pyr. POM-pyr was synthesized following the reported procedure. ${ }^{31}$

Synthesis of SWCNT-POM hybrid. $6 \mathrm{mg}$ of SWCNTs were dispersed in $24 \mathrm{~mL}$ of DMF by applying ultrasound for 30 minutes (Brandsonic 5510). Afterwards, $8 \mathrm{mg}$ of POM-pyr were added to the above mixture and the slurry stirred for 4 hours. Then, the mixture was left to settle down for one night, and the nanotubes were washed by dispersing them first in DMF and secondly in acetonitrile with the aid of ultrasound. Finally, SWCNT-POM were isolated by filtering through a nylon membrane filter $(0.2 \mu \mathrm{m}$ pore size $)$ and washing them with acetonitrile and with ethanol.

\section{Physical characterization}

HR-TEM images were obtained using a TECNAI G2 F20 microscope. Field Emission Gun (FEG) $200 \mathrm{kV}$. Aberration corrected HAADF and BF-STEM images were obtained in a JEOL ARM $200 \mathrm{~F}$ microscope operating at $80 \mathrm{keV}$, equipped with CEOS spherical aberration correctors both for probe and image formation. Spherical aberration coefficients C3 is almost $0 \mathrm{~mm}$ and $\mathrm{C} 5$ is around $5 \mathrm{~mm}$. An objective aperture has been used, and the convergence semiangle is $22 \mathrm{mrad}$. Inner and outer annular detector angles for HAADF STEM are about 50 and 200 mrad, respectively. Thermogravimetric analysis was carried out with a Mettler Toledo TGA/SDTA 851 apparatus in the $25-800^{\circ} \mathrm{C}$ temperature range under an air atmosphere and at $10 \mathrm{~K} \mathrm{~min}^{-1}$ scan rate. X-Ray photoelectron spectroscopy (XPS) measurements were performed in an ultra-high vacuum system ESCALAB210 (base pressure $1.0 \times 10^{-10}$ mbar) from Thermo VG Scientific. Photoelectrons were excited by using the $\mathrm{Mg}-\mathrm{K} \alpha$ line $(1253.6 \mathrm{eV})$. All spectra have been referred to the Fermi level.

Photophysical measurements. Steady-state UV/vis/NIR absorption spectroscopy was performed on a Cary 5000 spectrometer (Varian). Transient absorption spectroscopy was performed with $387 \mathrm{~nm}$ laser pulses from an amplified Ti/sapphire laser system (ModelCPA2101, Clark-MXR Inc.; output: $775 \mathrm{~nm}$, $1 \mathrm{kHz}$, and $150 \mathrm{fs}$ pulse width) in the TAPPS (transient absorption pump/probe system) Helios from Ultrafast Systems with $200 \mathrm{~nJ}$ laser energy. Steady-state fluorescence spectra were taken from samples with a FluoroLog3 spectrometer (Horiba) with a IGA Symphony $(51211 \mu \mathrm{m})$ detector in the NIR detection range. The Raman spectra were recorded with a FT-Raman spectrometer RFS100 (Bruker).

Spectroelectrochemical measurements. All experiments were carried out in deoxygenated DMF purchased from Sigma Aldrich with $0.1 \mathrm{M}$ or $0.2 \mathrm{M} \mathrm{TBAPF}_{6}$ (Sigma Aldrich) as supporting electrolyte. All chemicals were used without further purification.

For spectroelectrochemical measurements, a quartz cell with an optical pathway of $1 \mathrm{~mm}$ or a home-made three neck cell was used in combination with a three electrode setup. A platinum 
gauze served as the working electrode, a platinum wire as the counter electrode and $\mathrm{Ag} / \mathrm{AgNO}_{3}$ in acetonitrile was chosen as the reference electrode. In the case of the three neck cell, $\mathrm{Ag} /$ $\mathrm{AgNO}_{3}$ was replaced by a silver wire serving as a quasi reference electrode. Changes in absorption were monitored either by a Specord S600 (Analytik Jena) spectrometer or a Cary 5000 spectrometer (Varian).

Potentials were applied and controlled by means of a PGSTAT 101 potentiostat from Methrom $($ ) and the corresponding software NOVA 1.6@.

\section{Conclusions}

Pyrene-appended Dawson-Wells-type polyoxometalates have been successfully immobilized onto SWCNTs by means of noncovalent forces. Non-covalent functionalization relies on the presence of $\pi-\pi$ interactions between the sidewalls of SWCNTs and the $\pi$-conjugated pyrenes, which afford stable suspensions of strongly coupled SWCNT-POM hybrids. Formation of the hybrids was confirmed with high-resolution electron microscopy, XPS experiments, and thermogravimetric analysis. From the latter we estimate a loading of at least 1 POM-pyr per 4600 carbon atoms, which corresponds to a $10 \%$ coverage. Our photophysical studies confirm electron transfer from the photoexcited SWCNTs to the grafted polyoxometalate. The dynamics of the process, studied by transient absorption spectroscopy, reveal the appearance of metastable characteristics of oxidized SWCNTs and reduced POM-pyr. This is quite remarkable, as both components, POM-pyr and SWCNTs, are commonly used as electron acceptors. Our results demonstrate the potential of SWCNTs and POM-pyr for the design of photoactive molecular devices, which alongside the ability of POMs to undergo multiple reduction reactions with minor structural changes, qualify them as promising electron reservoirs for the design of advanced dyads.

\section{Abbreviations}

$\begin{array}{ll}\text { POMs } & \text { Polyoxometalates } \\ \text { SWCNTs } & \text { Single walled carbon nanotubes } \\ \text { FETs } & \text { Field-effect transistors } \\ \text { Pyr } & \text { Pyrene } \\ \text { TGA } & \text { Thermogravimetric analysis } \\ \text { EDS } & \text { Energy-dispersive X-ray spectroscopy } \\ \text { HRTEM } & \text { High resolution transmission electron microscopy } \\ \text { HAADF- } & \text { High-angle annular dark-field scanning- } \\ \text { STEM } & \text { transmission electron microscopy } \\ \text { BF } & \text { Bright field } \\ \text { XPS } & \text { X-Ray photoelectron spectroscopy } \\ \text { BEs } & \text { Binding energies }\end{array}$

\section{Acknowledgements}

Financial support from the EU (Projects COST Action CM1203 PoCHeMoN, ELFOS and ERC Advanced Grant SPINMOL), the
Spanish Ministerio de Economía y Competitividad MINECO (Project MAT2011-22785), the Generalitat Valenciana (Prometeo Program) and the Bayrische Staatsregierung as part of the "Solar Technologies go Hybrid" are gratefully acknowledged. AMR and SIM acknowledge the support from the Spanish MINECO (projects TEC2011-29120-C05-03 and Consolider Ingenio 2010 CSD2009-00013) and the Junta de Andalucía (PAI research group TEP-946 INNANOMAT), and also to JEOL company for the use of a double-corrected aberration-corrected ARM electron microscope. C.M.-G. thanks the Spanish MINECO for a Ramón y Cajal Fellowship (RYC-2012-10894).

\section{Notes and references}

1 D. Eder, Chem. Rev., 2010, 110, 1348.

2 R. H. Baughman, A. A. Zakhidov and W. A. de Heer, Science, 2002, 297, 787.

3 M. Urdampilleta, S. Klyatskaya, J.-P. Cleuziou, M. Ruben and W. Wernsdorfer, Nat. Mater., 2011, 10, 502; L. Bogani and W. Wernsdorfer, Inorg. Chim. Acta, 2008, 361, 3807.

4 V. Sgobba and D. M. Guldi, Chem. Soc. Rev., 2009, 38, 165. 5 Y. Liang, Y. Li, H. Wang and H. Dai, J. Am. Chem. Soc., 2013, 135, 2013; F. Li, B. Zhang, X. Li, Y. Jiang, L. Chen, Y. Li and L. Sun, Angew. Chem., 2011, 123, 12484.

6 C. Dekker, S. J. Tans and A. R. M. Verschueren, Nature, 1998, 393, 49.

7 J. Kong, N. R. Franklin, C. Zhou, M. G. Chapline, S. Peng, K. Cho and H. Dai, Science, 2000, 287, 622; E. Katz and I. Willner, ChemPhysChem, 2004, 5, 1084.

8 D.-L. Long, E. Burkholder and L. Cronin, Chem. Soc. Rev., 2007, 36, 105.

9 M. A. AlDamen, J. M. Clemente-Juan, E. Coronado, C. MartíGastaldo and A. Gaita-Ariño, J. Am. Chem. Soc., 2008, 130, 8874.

10 L. Bogani and W. Wernsdorfer, Nat. Mater., 2008, 7, 179; J. M. Clemente-Juan, E. Coronado and A. Gaita-Ariño, Chem. Soc. Rev., 2012, 41, 7464.

11 A. Sartorel, M. Carraro, G. Scorrano, R. De Zorzi, S. Geremia, N. D. McDaniel, S. Bernhard and M. Bonchio, J. Am. Chem. Soc., 2008, 130, 5006; Y. V. Geletii, B. Botar, P. Kögerler, D. A. Hillesheim, D. G. Musaev and C. L. Hill, Angew. Chem., 2008, 120, 3960; S. Goberna-Ferrón, L. Vigara, J. Soriano-López and J. R. G. Mascarós, Inorg. Chem., 2012, 51, 11707; H. Lv, Y. V. Geletii, C. Zhao, J. W. Vickers, G. Zhu, Z. Luo, J. Song, T. Lian, D. G. Musaev and C. L. Hill, Chem. Soc. Rev., 2012, 41, 7572.

12 D. Pan, J. Chen, W. Tao, L. Nie and S. Yao, Langmuir, 2006, 22, 5872 .

13 A. Giusti, G. Charron, S. Mazerat, J. D. Compain, P. Mialane, A. Dolbecq, E. Rivière, W. Wernsdorfer, R. Ngo Biboum, B. Keita, L. Nadjo, A. Filoramo, J.-P. Bourgoin and T. Mallah, Angew. Chem., Int. Ed., 2009, 48, 4949; G. Charron, A. Giusti, S. Mazerat, P. Mialane, A. Gloter, F. Miserque, B. Keita, L. Nadjo, A. Filoramo, E. Rivière, W. Wensdorfer, V. Huc, J.-P. Bourgoin and T. Mallah, Nanoscale, 2010, 2, 139. 
14 F. M. Toma, A. Sartorel, M. Iurlo, M. Carraro, P. Parisse, C. Maccato, S. Rapino, B. R. Gonzalez, H. Amenitsch, T. Da Ros, L. Casalis, A. Goldoni, M. Marcaccio, G. Scorrano, G. Scoles, F. Paolucci, M. Prato and M. Bonchio, Nat. Chem., 2010, 2, 826.

15 N. Kawasaki, H. Wang, R. Nakanishi, S. Hamanaka, R. Kitaura, H. Shinohara, T. Yokoyama, H. Yoshikawa and K. Awaga, Angew. Chem., Int. Ed., 2011, 50, 3471; H. Wang, N. Kawasaki, T. Yokoyama, H. Yoshikawa and K. Awaga, Dalton Trans., 2012, 41, 9863.

16 B. Matt, C. Coudret, C. Viala, D. Jouvenot, F. Loiseau, G. Izzet and A. Proust, Inorg. Chem., 2011, 50, 7761; B. Matt, X. Xiang, A. L. Kaledin, N. Han, J. Moussa, H. Amouri, S. Alves, C. L. Hill, T. Lian, D. G. Musaev, G. Izzet and A. Proust, Chem. Sci., 2013, 4, 1737; B. Matt, J. Fize, J. Moussa, H. Amouri, A. Pereira, V. Artero, G. Izzet and A. Proust, Energy Environ. Sci., 2013, 6, 1504.

17 G. Izzet, M. Ménand, B. Matt, S. Renaudineau, L.-M. Chamoreau, M. Sollogoub and A. Proust, Angew. Chem., Int. Ed., 2012, 51, 487.

18 C. Rinfray, G. Izzet, J. Pinson, S. Gam Derouich, J.-J. Ganem, C. Combellas, F. Kanoufi and A. Proust, Chem. - Eur. J., 2013, 19, 13838.

19 V. Duffort, R. Thouvenot, C. Afonso, G. Izzet and A. Proust, Chem. Commun., 2009, 6062; M. M. Lorion, B. Matt, S. Alves, A. Proust, G. Poli, J. Oble and G. Izzet, Chem. Eur. J., 2013, 19, 12607.

20 A. Proust, B. Matt, R. Villanneau, G. Guillemot, P. Gouzerh and G. Izzet, Chem. Soc. Rev., 2012, 41, 7605.

21 M. Herranz, N. Martín, S. Campidelli, M. Prato, G. Brehm and D. M. Guldi, Angew. Chem., 2006, 118, 4590; B. Ballesteros, G. de L. Torre, C. Ehli, G. M. A. Rahman, F. Agulló-Rueda, D. M. Guldi and T. Torres, J. Am. Chem. Soc., 2007, 129, 5061; C. Oelsner, M. A. Herrero, C. Ehli, M. Prato and D. M. Guldi, J. Am. Chem. Soc., 2011, 133, 18696; C. Bosch-Navarro, E. Coronado, C. Martí-Gastaldo, B. Rodríguez-González and L. M. Liz-Marzán, Adv. Funct. Mater., 2012, 22, 979.

22 E. W. McQueen and J. I. Goldsmith, J. Am. Chem. Soc., 2009, 131, 17554.

23 S. Kyatskaya, J. R. G. Mascarós, L. Bogani, F. Hennrich, M. Kappes, W. Wernsdorfer and M. Ruben, J. Am. Chem. Soc., 2009, 131, 15143; L. Bogani, C. Danieli, E. Biavardi, N. Bendiab, A.-L. Barra, E. Dalcanale, W. Wernsdorfer and
A. Cornia, Angew. Chem., Int. Ed., 2009, 48, 746; M. Ganzhorn, S. Klyatskaya, M. Ruben and W. Wernsdorfer, ACS Nano, 2013, 7, 6225.

24 J. Bartelmess, C. Ehli, J.-J. Cid, M. García-Iglesias, P. Vázquez, T. Torres and D. M. Guldi, J. Mater. Chem., 2011, 21, 8014; M. Ince, J. Bartelmess, D. Kiessling, K. Dirian, M. V. Martínez-Díaz, T. Torres and D. M. Guldi, Chem. Sci., 2012, 3, 1472.

25 C. Schulz Drost, V. Sgobba, C. Gerhards, S. Leubner, R. M. Krick Calderon, A. Ruland and D. M. Guldi, Angew. Chem., Int. Ed., 2010, 49, 6425; L. Hu, Y.-L. Zhao, K. Ryu, C. Zhou, J. F. Stoddart and G. Grüner, Adv. Mater., 2008, 20, 939.

26 H. Xin and A. T. Woolley, J. Am. Chem. Soc., 2003, 125, 8710. 27 C. Romero-Nieto, R. García, M. A. Herranz, C. Ehli, M. Ruppert, A. Hirsch, D. M. Guldi and N. Martin, J. Am. Chem. Soc., 2012, 134, 9183.

28 D. Ma, L. Liang, W. Chen, H. Liu and Y.-F. Song, Adv. Funct. Mater., 2013, 23, 6100; G. Modugno, Z. Syrgiannis, A. Bonasera, M. Carraro, G. Giancane, L. Valli, M. Bonchio and M. Prato, Chem. Commun., 2014, 50, 4881.

29 B. Dawson, Acta Crystallogr., 1953, 6, 113.

30 D.-L. Long, P. Kögerler, A. D. C. Parenty, J. Fielden and L. Cronin, Angew. Chem., Int. Ed., 2006, 45, 4798; L. VilàNadal, K. Peuntinger, C. Busche, J. Yan, D. Lüders, D.-L. Long, J. M. Poblet, D. M. Guldi and L. Cronin, Angew. Chem., Int. Ed., 2013, 52, 9695.

31 B. Matt, S. Renaudineau, L. M. Chamoreau, C. Afonso, G. Izzet and A. Proust, J. Org. Chem., 2011, 76, 3107.

32 D. Bom, R. Andrews, D. Jacques, J. Anthony, B. Chen, M. S. Meier and J. P. Selegue, Nano Lett., 2002, 2, 615.

33 X. Zhang, L. Hou, A. Cnossen, A. C. Coleman, O. Ivashenko, P. Rudolf, B. J. van Wees, W. R. Browne and B. L. Feringa, Chem. - Eur. J., 2011, 17, 8957.

34 R. Liu, S. Li, X. Yu, G. Zhang, S. Zhang, J. Yao and L. Zhi, J. Mater. Chem., 2012, 22, 3319.

35 Changes due to differences in the environment are, however, ruled out based on the similarities in absorption features.

36 H. Kataura, Y. Kumazawa, Y. Maniwa, I. Umezu, S. Suzuki, Y. Ontsuka and Y. Achiba, Synth. Met., 1999, 103, 2555.

37 C. Romero-Nieto, R. Garcia, M. Angeles-Herranz, L. Rodriguez-Perez, M. Sanchez-Navarro, J. Rojo, N. Martin and D. M. Guldi, Angew. Chem., Int. Ed., 2013, 52, 10216. 